\title{
Determinants of noise level in the neonatal intensive care unit at Castle Street Hospital for Women, Colombo, a level III tertiary care facility
}

\author{
*Chamara Jayamanne ${ }^{1}$, Saman Kumara ${ }^{2}$ \\ Sri Lanka Journal of Child Health, 2019; 48(4): 312-315
}

\begin{abstract}
Introduction: Advances in modern neonatology have improved survival of extremely premature and extremely low birth weight babies. Increased noise level is proven to be harmful to preterm babies and term ill babies in various ways. Determinants and baseline noise level should be assessed before necessary intervention.
\end{abstract}

Objective: To assess the determinants of noise level at a level III neonatal intensive care unit (NICU) at the Castle Street Hospital for Women (CSHW), Sri Lanka.

Method: A prospective observational study was done at NICU, CSHW, Sri Lanka. Data were collected using two NDI USB Digital sound level data loggers that recorded noise level on a weighted decibel scale (dBA) every minute continuously for three weeks. Data was exported using provided software. Average noise level was analysed separately for consultant ward round (9am-12pm), nursing handing-over time (Daily 77.30am, 1-1.30pm and 7-7.30pm), admissions/emergencies (time taken from records) and a period supposed to be silent (Daily 1am$3 \mathrm{am}$ ). Recommended standard ambient noise level for NICU by the American Academy of Paediatrics as well as Australasian Health Infrastructure Alliance is less than $45 \mathrm{~dB}$.

Results: Average noise level recorded in NICU was $60 \mathrm{dBA}$ which is considerably higher than recommended levels. During the consultant ward round the average noise level was $60.2 \mathrm{dBA}$. Noise levels at the morning, afternoon and evening

${ }^{1}$ Senior Registrar in Paediatrics, Lady Ridgeway Hospital for Children, Colombo, Sri Lanka, ${ }^{2}$ Consultant Neonatologist, Castle Street Hospital for Women, Colombo, Sri Lanka

*Correspondence: chamarajy@gmail.com

orcid.org/ 0000-0001-8256-6021

(Received on 18 February 2019: Accepted after revision on 22 March 2019)

The authors declare that there are no conflicts of interest

Personal funding was used for the project.

Open Access Article published under the Creative

Commons Attribution CC-BY (c) (P) handing-over times were 62.9, 62.7 and $60.9 \mathrm{dBA}$ respectively. Noise level at the supposed silent period (Daily 1am-3am) was $56.8 \mathrm{dBA}$. During admissions or emergencies which required ventilation (CPAP/conventional), the average noise level was as high as $65.8 \mathrm{dBA}$.

Conclusions: Noise levels recorded in the NICU at the CSHW, Sri Lanka in all circumstances were higher than the recommended noise levels. Main contributions were machine alarms and conversations.

DOI: http://dx.doi.org/10.4038/sljch.v48i4.8824

(Key words: Noise level determinants, level III care, Neonatal Intensive Care Unit, noise level data loggers, quality newborn care)

\section{Introduction}

Advances in modern neonatal care have improved the survival and outcome of high risk preterm and extremely low birth weight babies over the past few decades ${ }^{1}$. The number of preterm deliveries has also increased due to better antenatal fetal monitoring compared to the past ${ }^{2}$. With advancement of care for extremely premature and extremely low birth weight babies in Sri Lanka, it is time for us to improve the quality of developmental care in the neonatal intensive care units (NICUs) ${ }^{3}$.

Studies have shown that for the fast recovery and wellbeing of the patients, health care environment plays a major role ${ }^{4}$. Premature babies lose their protective environment in utero thus exposing themselves to the external environment including the central auditory organs ${ }^{5}$. Further, many researchers have shown that loud noises are a primary stressor for ill preterm neonates as well as for the staff working in the environment ${ }^{6}$.

Even term neonates who are sick lose their ability to filter noxious stimuli so that they are vulnerable to loud noises which can still harm the auditory system $^{6}$. In utero fetuses only receive expected low frequency high quality sounds through amniotic fluid medium. However, in a NICU they will receive high frequency unexpected noises from ventilator alarms, fans, monitors, infusion pumps, voices, banging incubator porthole doors etc. through air medium. In preterm neonates this 
difference (acoustic gap) may harm the smooth development of the auditory system ${ }^{7}$. It can be as harmful as causing an intracranial haemorrhage ${ }^{8}$. These effects later on will lead to development of auditory, language and attentive problems in childhood ${ }^{9}$.

It has been shown that aminoglycoside administration in the background of loud noises can potentiate reactions which can increase the ototoxicity $^{10}$. Loud noises at NICU can affect cardiovascular and respiratory instability and cause sleep deprivation. The effect on neurodevelopmental outcome is still to be determined $^{9}$. Similar results were obtained by several other researchers who found that loud noises increase heart rate and respiratory rate and decreased peripheral oxygen saturation ${ }^{10}$. Exposure to loud noises for more than 48 hours is considered a risk factor for hearing loss ${ }^{10}$.

It has also been shown that preterm newborns exposed to loud noises take a long time to achieve weight and they have a prolonged NICU stay ${ }^{11}$. Committee on environmental hazards of American Academy of Paediatrics has concluded that increased noise in NICU will cause detrimental side effects to extreme premature as well as sick neonates. It clearly says loud noise may result in cochlear damage and may disrupt the normal growth and development ${ }^{6}$.

Thus, having a desired noise level in a NICU is important. Recommended standard ambient noise level for NICU is less than $45 \mathrm{~dB}$ by the American Academy of Paediatrics as well as Australasian Health Infrastructure Alliance, 2013 $3^{6,12}$. However, studies have shown that in many NICUs sound level exceeds that of recommended level in various ways ${ }^{13}$. Often, staff are not aware how loud the surrounding noises actually are ${ }^{14}$. There are neither published data, to the best of our knowledge, in Sri Lanka with regards to the average noise level and causes for the increased noise level nor any research being carried out to optimise the noise level in NICUs.

\section{Objectives}

To assess the determinants of noise level at a level III NICU at the Castle Street Hospital for Women (CSHW), Sri Lanka.

\section{Method}

An observational prospective study was carried out in CSHW which is one of the largest tertiary care maternity hospitals in Sri Lanka. The CSHW NICU is recognized as an institute with level III care ${ }^{16}$. It provides care to extremely premature neonates and term sick newborns.

The NICU is $1680 \mathrm{~m}^{2}$ large and it consists of 6 beds as Intensive Care Unit and other 5 beds as High Dependency Unit (Figure 1) NICU equipped with 7 ventilators including 1 high frequency oscillatory ventilator, 3 CPAP machines, High flow Oxygen meters, multi-para monitors for every bed and 4 infusion pumps per each bed with incubators/open warm cots, suction apparatus and phototherapy units. NICU can perform routine procedures done in all level III NICU including intubation, arterial and venous line insertion, X-ray facility, ultrasound and $2 \mathrm{D}$ echo facilities, emergency resuscitation and so on. NICU phone is kept in the nurses' station.

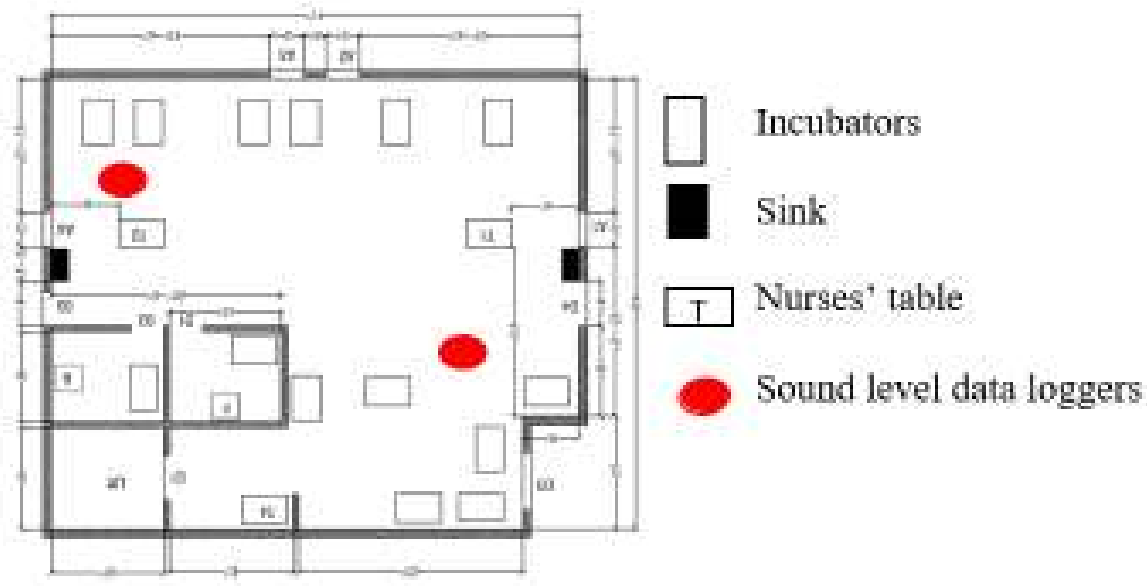

Figure 1: Neonatal intensive care unit

Consultant ward rounds were done every day inside the unit 9 am to $12 \mathrm{pm}$ and nursing handing over was done 7.00 am to $7.30 \mathrm{am}, 1.00 \mathrm{pm}$ to $1.30 \mathrm{pm}$ and $7.00 \mathrm{pm}$ to $7.30 \mathrm{pm}$ every day. Noise level data collection was done using two digital sound level data loggers fixed in NICU as depicted in Figure 1 which continuously recorded noise level each minute noise level along with the date and the 
time of recording. It had a battery life span of 6 months even with continuous use. Data loggers were fixed 2 feet below the ceiling and 8 feet above the ground. Study was conducted for a period of three weeks starting from December 2016 after the approval of relevant authorities. The noise level was calculated as an average for the period of three weeks and compared with the standard recommended levels. Average noise levels were analysed separately for consultant ward round (9am-12pm), nursing handing-over time (Daily 7$7.30 \mathrm{am}, 1-1.30 \mathrm{pm}$ and $7-7.30 \mathrm{pm})$, admissions/ emergencies (time taken from records) and a period supposed to be silent (Daily 1am-3am).

Ethical clearance and consent, and confidentiality of data: Approval was taken from the Ethics Review Committee of the CSHW prior to conducting this research. Informed permission was taken from the Director of the Hospital. All the staff members including doctors, nurses and attendant staff were informed regarding the noise level data logger recordings and it was emphasized that it will not record individual voices but only the noise level.

\section{Results}

Average noise level recorded in NICU was 60 dBA. During consultant ward round average noise level was $60.2 \mathrm{dBA}$. Morning, afternoon and evening handing-over time, noise levels were 62.9, 62.7 and $60.9 \mathrm{dBA}$ respectively. Noise level at supposed silent period (Daily 1am-3am) was 56.8dBA. There were 15 admissions during the study period, 8 needed CPAP and 7 needed conventional ventilation. There were 2 medical emergencies. Analysis of those instances revealed an average noise level of $65.8 \mathrm{dBA}$.

\section{Discussion}

The average noise level recorded in the NICU was 60dBA which is considerably higher than the recommended standard ambient noise level for NICU by the American Academy of Paediatrics and Australasian Health Infrastructure Alliance which is less than $45 \mathrm{dBA}^{12,16}$. The morning, afternoon and evening handing-over time noise levels were $62.9,62.7$ and $60.9 \mathrm{dBA}$ respectively indicating contribution of human voices to noise level. Noise level at the supposed silent period (1am-3am daily) was 56.8dBA indicating contribution of alarms and machines. This study clearly shows that the average noise level of the NICU exceeds recommended ambient noise level for a NICU. Main contributors are human voices, alarms, machines and various emergencies which increase the voice rather due to the anxiety of the medical professionals in response to the situation. This needs to be addressed to provide better environment for these babies. As the first step it is imperative to conduct educational sessions mentioning the value of controlling noise for the whole team taking care of ill and preterm babies representing all the levels of professionals. A noise level policy can be introduced so that the recommended levels can be achieved slowly in stepwise pattern. Noise level alert meter indicating noise level and posters reminding the noise level policy may be useful in achieving the desirable noise level which in turns increases the quality of newborn care.

\section{Conclusions}

Noise levels recorded in the NICU at the Castle Street Hospital for Women, Sri Lanka in all circumstances were higher than the recommended noise levels. Main contributions were machine alarms and conversations.

\section{References}

1. Stoelhorst GMSJ, Rijken M, Martens SE, Brand R, den Ouden AL, Wit J-M, et al. Changes in neonatology: Comparison of two cohorts of very preterm infants (Gestational age \& amp; lt; 32 weeks): The project on preterm and small for gestational age infants 1983 and the Leiden follow-up project on prematurity 1996-1997. Pediatrics [Internet]. 2005 Feb 1; 115(2):396 LP-405. Available from: http://pediatrics.aappublications.org/conte nt/115/2/396.abstract

2. Martin J, Hamilton BE, Sutton PD, Ventura SJ, Menacker F, Kirmeyer S, et al. Births : Final Data for 2006. National Vital Statistics Reports 2009; 57(7):1-104.

3. Amarasena S. Position paper of the Sri Lanka College of Paediatricians on resuscitation of extremely premature / low birth weight newborns. Sri Lanka Journal of Child Health 2015; 44(1):3-11. https://doi.org/10.4038/sljch.v44i1.7956

4. Lawson B, Phiri M. The Architectural Healthcare Environment and its Effects on Patient Health Outcomes. 2013; (March):27.

5. Graven S. Sleep and Brain Development. Clinical Perinatology 2016; 33(3):693706.

https://doi.org/10.1016/j.clp.2006.06.009

6. Health E. Committee on Environmental Health Noise : A Hazard for the Fetus and Newborn. 1997;100(4). 
7. Lahav A, Skoe E. An acoustic gap between the NICU and womb: A potential risk for compromised neuroplasticity of the auditory system in preterm infants. Frontiers in Neuroscience 2014; 8:1-8. https://doi.org/10.3389/fnins.2014.00381

8. Manuscript A. NIH Public Access. 2011; 67(1):1-8.

9. Wachman EM, Lahav A. The effects of noise on preterm infants in the NICU. Archives of Disease in Childhood. Fetal Neonatal Edition 2011;96(4):F305-9. https://doi.org/10.1136/adc.2009.182014

10. Zimmerman E, Lahav A. Ototoxicity in preterm infants: effects of genetics, aminoglycosides, and loud environmental noise. Journal of Perinatology 2013; 33(1): 3-8.

https://doi.org/10.1038/jp.2012.105

11. Kamlin COF, O’Donnell CPF, Everest NJ, Davis PG, Morley CJ. Accuracy of clinical assessment of infant heart rate in the delivery room. Resuscitation 2006; 71(3):319-21.

https://doi.org/10.1016/j.resuscitation.200 6.04 .015

12. Health A, Alliance I. Australasian Health Facility Guidelines Part D - Infection Prevention and Control. 2015;(June).
13. Krueger C, Schue S, Parker L. Neonatal intensive care unit sound levels before and after structural reconstruction. American Journal of Maternal/ Child Nursing 2007; 32(6):358-62. https://doi.org/10.1097/01.NMC.0000298 131.55032 .76

14. Darcy AE, Hancock LE, Ware EJ. A descriptive study of noise in the neonatal intensive care unit: ambient levels and perceptions of contributing factors. Advanced Neonatal Care 2008; 8(5 Suppl):S16-26. https://doi.org/10.1097/01.ANC.00003372 $68.85717 .7 \mathrm{c}$

15. Wang D, Aubertin C, Barrowman N, Moreau K, Dunn S, Harrold J. Reduction of noise in the neonatal intensive care unit using sound-activated noise meters. Archives of Disease in Childhood Fetal Neonatal Edition 2014; 99(6):F515-6. https://doi.org/10.1136/archdischild-2014306490

16. American Academy of Pediatrics. Policy Statement. Levels of Neonatal Care. Pediatrics 2012;130(3):587-97. https://doi.org/10.1542/peds.2012-1999 